\title{
Zahntechnische Materialien: Welche Auslage wird ersetzt?
}

\author{
Die Kassenzahnärztliche Bundesvereinigung (KZBV) \\ und die Bundeszahnärztekammer (BZÄK) haben im \\ Oktober 2015 die 1. Auflage einer gemeinsamen Bro- \\ schüre zum „Einkauf von Materialien - Rechtsgrund- \\ lagen und Hinweise für die Zahnarztpraxis" \\ herausgegeben. Darin steht, welche begleitenden \\ Materialkosten privat abrechnungsfähig sind.
}

Einleitendes Zitat der Broschüre: „Neben einer Reihe von Nebenpflichten übernimmt der Zahnarzt mit dem Behandlungsvertrag vor allem die Verpflichtung zur Erbringung der Heilbehandlung, einschließlich der Beschaffung aller hierfür erforderlichen Materialien, Werkstücke usw. - vom Wattetupfer über Füllungsmaterialien bis zum Zahnersatz.

Ob und in welchem Umfang die zu diesem Zweck beschafften Materialien dem Patienten gesondert in Rechnung gestellt werden können oder mit dem zahnärztlichen Honorar abgegolten sind, ist - abhängig vom Material - unterschiedlich geregelt.“ (Siehe umfassende Übersichten unter $w w w$.alex-za.de „Verbrauchsmaterialien“.)

\section{Materialien gemäß § 9 GOZ („private“ zahntechnische Materialien)}

Zuzüglich zu angefallenen Herstellungskosten, zu sogenannten „Laborkosten“ zur zahntechnischen Erstellung von Werkstücken und Hilfsmitteln, sind gegebenenfalls auch zahntechnische Chairside-Herstellung begleitende Materialkosten privat berechnungsfähig, zum Beispiel

- Abdämmmassen (Stempelmaterial, Stopps etc.)

- Aufbissbehelfe, konfektionierte (Fertigteile zur Individualisierung)

- Ausblockmaterial (nicht für intraorale Aufbaufüllungen)

- Dubliermassen (Silikone, Alginate etc.)

- Einschleifmittel (keine wieder verwendbaren Instrumente)

- Form-Fertigteile (Hülsen, Kappen, Ringe, Manschetten, Aufbauhilfsteile) usw.

Es gibt die These, da von dem Labor die dauerhaft im Mund verbleibenden Materialien nach dem Medizinproduktegesetz mit der Konformitätserklärung ausgewiesen werden müssen, seien diese auch gesondert berechnungsfähig. Daher könnten im privaten Behandlungsvertrag weitere Fertigungsmaterialien berechnet werden wie

- Kompositverblendmaterial

- Keramikverblendmaterial

- Prothesen- bzw. Prothesenreparaturkunststoffe

- Modellgussmaterial

- technische Fertigteile wie Sockelplatten

- Keramikrohlinge bzw. Grundmaterial je Einheit (Fräs- oder Presstechnik)

\section{Labormaterialien gemäß BEL II (GKV, auch „privat" ansetzbar)}

Berechnungsfähig gemäß $₫ 4$ des BEL II sind die Kosten für Sonder- oder Weichkunststoffe

- Konfektionsfertigteile (z. B. Brückenteilungsgeschiebe)

- Implantate (zahntechnische) bzw.

- Implantataufbauten ggf. mit Implantat-bedingten Verbindungselementen

- Registrierbesteck (Verbrauchsteile) zur Stützstiftregistrierung

- Ersatzzähne, konfektioniert

- edelmetallhaltige Dentallegierungen (Lotkosten je Stelle bei Instandsetzung/Erweiterung)

\section{Materialkalkulation}

Das „gewerbliche Labor“ (Fremdlabor) und das sogenannte „Praxislabor" (Eigenlabor) - grundsätzlich auch ein gewerbliches Labor - kalkulieren die Materialberechnung nach allgemein gültigen betriebswirtschaftlichen Grundsätzen. Die zahnärztliche Rechnung im privaten Behandlungsvertrag dokumentiert die tatsächlich entstandenen angemessenen Materialkosten gemäß $\$ 9$ GOZ (natürlich in erster Linie die Herstellungskosten, zum Beispiel nach BEB) regelmäßig mit einem Rechnungsanhang in Form einer „Rechnung des Dentallabors“, in aller Regel auf der Basis einer grundsätzlich vereinbarten Laborkosten-Preisliste.

Gemäß $\$ 4$ Abs. 3 GOZ sind nur Praxiskosten, keinerlei Material- und Laborkosten abgegolten: Unter abgegoltene Praxiskosten werden insbesondere die Kosten für „Füllungsmaterial“ für den „Sprechstundenbedarf“, für die (behandlungsbegleitende) „Anwendung von Instrumenten und Apparaten“ sowie für „Lagerhaltung“ eingeordnet.

Selbstverständlich kann ein gewerbliches Fremd- oder Eigenlabor nicht lediglich Herstellungskosten deckend seine Preise kalkulieren, sondern es muss zuzüglich weitere tatsächlich anfallende Kosten wie Ausfall- und Gewährleistungsrisiko, Vorfinanzierungsentgelt oder Aufbewahrungs-/Lagerungskosten etc. und einen angemessenen Gewinnaufschlag" (amtliche Begründung der Bundesregierung, 1987) berücksichtigen.

Interessant für die Rechnungserstellung/-prüfung ist eine Aufstellung, bei welchen zahnmedizinischen Leistungen welche Materialkosten nach $₫ 9 \mathrm{GOZ}$ anfallen können.

(c) $Z A ; P . E$.

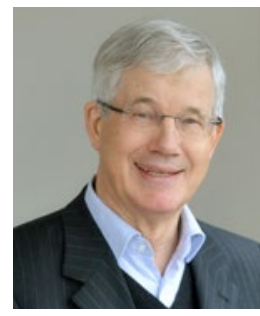

Dr. Peter H.G. Esser

GOZ-Experte und -Berater der ZA eG www.alex-za.de 